\title{
Pairing Symmetry, Phase diagram and Edge Modes in Topological Fulde-Ferrell-Larkin-Ovchinnikov Phase
}

\author{
Chun Fai Chan and Ming Gong* \\ Department of Physics and Centre for Quantum Coherence, \\ The Chinese University of Hong Kong, Shatin, N.T., Hong Kong, China
}

\begin{abstract}
The realizations of spin-orbit coupling in cold atoms lead to a burst of research activities in the searching of topological matters in ultracold atom systems. The very recent theoretical predictions show that topological Fulde-Ferrell-Larkin-Ovchinnikov (FFLO) superfluids can be realized with proper spin-orbit coupling and Zeeman fields. In this work, a comprehensive understanding of the pairing symmetry, phase diagram and the edge modes in this new topological matter are presented. The momentum of the Cooper pairs plays the role of renormalizing the in-plane Zeeman field and chemical potential. The in-plane Zeeman field and finite momentum pairing induce asymmetry to the effective $p$-wave pairing, apart from a small fraction of higher orbital components. The phase diagram is composed by different phases, which are determined by the topology and band gap nature of the superfluids. Especially, the gapped and gapless topological FFLO phase have totally different finite size effect. These novel features show that the spin-orbit coupled cold atoms provides an important platform in realizing topological matters which may not be materialized with solids.
\end{abstract}

PACS numbers: 03.75.Ss, 74.20.Fg, 74.70.Tx, 03.67.Lx

The experimental realizations of spin-orbit coupling (SOC) in cold atom systems[1] 3] lead to a burst of research activities in the searching of novel new physics with this new interaction in the context of atomic, molecular and optical physics 4 24], among which the topological matters are probably the most widely pursued phenomena. This is not strange because SOC is always the most unusual interaction in condensed matter physics with nontrivial topology or geometry phase, see Ref. 25 27. Now it is widely recognized that the SOC and Zeeman field in degenerate Fermi gas can lead to topological Bardeen-Cooper-Schrieffer (BCS) phase when $h^{2}>\mu^{2}+\Delta^{2}$, where $h, \mu, \Delta$ are the Zeeman field strength (independent of its direction), chemical potential and order parameter, respectively. The basic reason is that SOC and Zeeman field can lead to equivalent $k_{x}+i k_{y}$ triplet pairing in the dressed basis (eigenvectors of the single particle Hamiltonian, see Fig. 10 under proper condition. This idea, first unveiled by Zhang 28] and Sato 29 in cold atoms, can be tracked back to the work by Gor'kov and Rashba 30], who showed that, due to inversion symmetry breaking induced SOC, the singlet and triplet pairing are mixed in the wave function of the Cooper pairs in non-centrosymmetric superconductors. It is then applied to solid materials to search Majorana Fermion, see the recent review and references therein [31]. This topological boundary put a strong constraint to the realization of Majorana Fermions in practical experiments because the Cooper pair may be destroyed by strong Zeeman field due to Pauli depairing effect. Moreover, in cold atom systems, the topological phase is only possible to be observed in the strong coupling regime [4]. So the realization of topological phase is always accompanied by competition with either Pauli depairing effect or strong coupling effect.
Generally speaking, there are two totally different paradigms to destroy the Cooper pair by strong Zeeman field. The most conventional way, for example, in type-I superconductors, is the direct destroying of Cooper pair via a second-order phase transition. The other paradigm is that the strong Zeeman field first induce spontaneous translation symmetry breaking to the uniform superconductor and then destroy it via a first order phase transition. The latter case may be encountered in some type-II superconductors where magnetic and superconducting order coexist, which is now widely known as the Fulde-Ferrell-Larkin-Ovchinnikov (FFLO) phase. Recent progresses show that the SOC and in-plane Zeeman field can provide an more efficient route 11, 12] to realize FFLO superfluids in ultracold atoms. The basic idea is quite simple, the in-plane Zeeman field can deform the Fermi surface, making the single particle bands lack inversion symmetry. As a result, it is impossible to find two fermions with opposite momentum but the same energy in the same band. Mathematically, we can write down the thermaldynamical potential as $\Omega(\mathbf{Q})=\Omega(0)+\nabla \Omega(0) \cdot \mathbf{Q}+\mathcal{O}\left(\mathbf{Q}^{2}\right)$ in the small $\mathbf{Q}$ limit, and we can proof exactly that $\nabla \Omega(0) \neq 0$ in the present of both in-plane Zeeman field and SOC 32]. The small $\mathbf{Q}$ will not change the topology of the Fermi surface, thus in some parameter regime the topological FFLO can be realized, as predicted in recent works [14 17]. From the language of topology, this new phase is just a deformed BCS phase. In this new phase, all the physical parameters are involved to determine the topological boundaries. Thus in principle, such a topological phase is a more suitable candidate for engineering topological phase transition in ultracold atoms.

The physics of this new phase is still not well understood. In this work, we aim to provide a comprehensive 


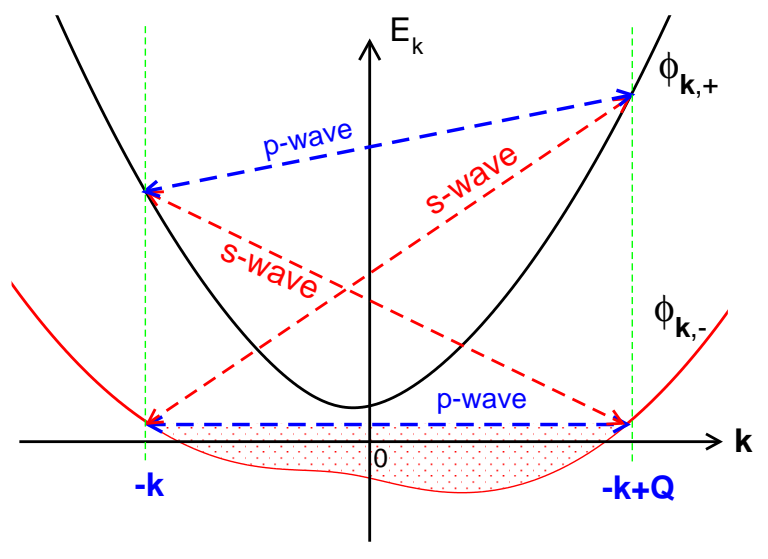

FIG. 1: (Color online). Single particle band structures with in-plane Zeeman field. The blue bidirectional arrows mark the possible $p$-wave pairing in the same band, and the red bidirectional arrows mark the $s$-wave pairing between different bands. The two different bands have different chirality $(s= \pm)$ or geometry phase 14, 28].

understanding of this new phase recently predicted in Ref. [14 17]. Our major observations are summarized as following: (I) The finite momentum of the Cooper pairs plays the role of renormalizing the in-plane Zeeman field and chemical potential. (II) The effective pairing in the topological FFLO phase regime is $p$-type plus a small fraction of higher orbital components. The effective in-plane Zeeman fields introduce asymmetry to the $p$-wave pairing, which can influence the properties of the superfluids. However, this asmmetry will not change the Fermi surface topology, which is only determined by the strength of the effective Zeeman field. (III) The phase diagram composed by different phases is determined by topology and band gap nature (gapped or gapless). The gapped and gapless topological phase have totally different finite size effect. These novel features in ultracold atom systems have no counterpart in solid materials.

In the past two years great theoretical endeavors have been made trying to understand the physics in spinorbital degenerate Fermi gas based on self-consistent calculations 4 24]. The triplet pairing induced by SOC is essential to overcome the Chandrasekhar-Clogston limit 33, 34], thus finite pairing can survive in the topological regime $\left(h^{2}>\Delta^{2}+\mu^{2}\right)$. The essential physics, however, can be well understood without these complicated calculations, therefore in the coming discussion, we mainly consider the following Bogliubov-de Gennes (BdG) Hamiltonian [11, 12, 14],

$$
H_{\mathrm{BdG}}(\mathbf{k})=\left(\begin{array}{cc}
H_{0}\left(\frac{\mathbf{Q}}{2}+\mathbf{k}\right) & \Delta \\
\Delta & -\sigma_{y} H_{0}^{*}\left(\frac{\mathbf{Q}}{2}-\mathbf{k}\right) \sigma_{y}
\end{array}\right),
$$

where the Nambu basis is chosen as $\left(c_{\mathbf{k}+\mathbf{Q} / 2, \uparrow}, c_{\mathbf{k}+\mathbf{Q} / 2, \downarrow}, c_{-\mathbf{k}+\mathbf{Q} / 2, \downarrow}^{\dagger},-c_{-\mathbf{k}+\mathbf{Q} / 2, \uparrow}^{\dagger}\right)^{T}, \quad$ with $c_{\mathbf{k} s}$ the destructive operator and $\mathbf{Q}=\left(Q_{x}, Q_{y}\right)$ the
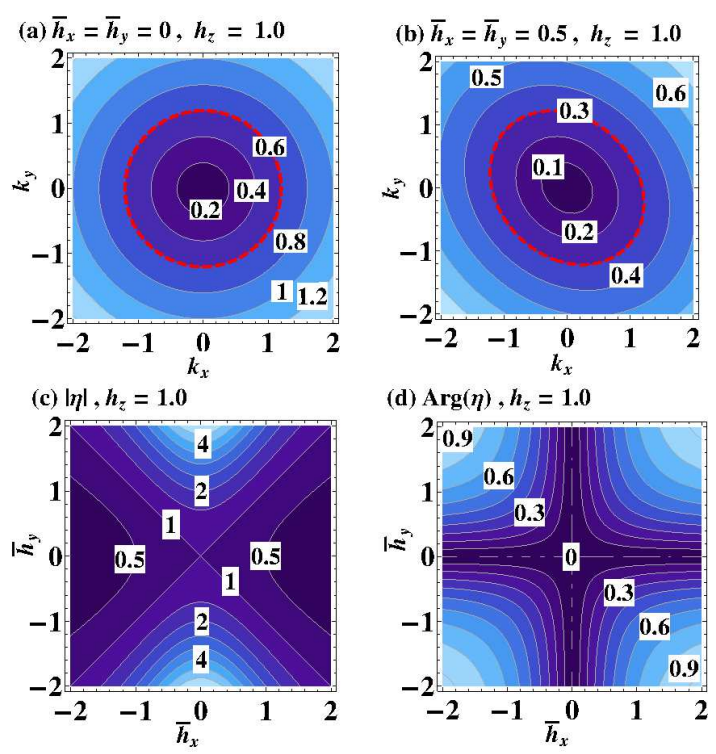

FIG. 2: (Color online). (a)-(b) the pairing function $\left|A_{\mathbf{k}, \mathbf{Q}}\right|$ in momentum space. (c)-(d) the absolute value and phase (Arg) of $\eta$ as a function of in-plane Zeeman field.

total Cooper pair momentum. $H_{0}$ is the single particle Hamiltonian,

$$
H_{0}=\mathbf{k}^{2}+\alpha\left(k_{x} \sigma_{y}-k_{y} \sigma_{x}\right)+h_{z} \sigma_{z}+h_{x} \sigma_{x}+h_{y} \sigma_{y},
$$

where $\mathbf{k}=\left(k_{x}, k_{y}\right), \alpha$ is the Rashba SOC coefficient, and $h_{x}, h_{y}, h_{z}$ are Zeeman field strength, and $\sigma_{x}, \sigma_{y}$ and $\sigma_{z}$ are Pauli matrices. Notice that the energy is scaled by Fermi energy $E_{\mathrm{F}}$, and the momentum is scaled by the corresponding Fermi momentum $k_{\mathrm{F}}$. This model can be readily realized with degenerate Fermi gas with Rashba SOC. Notice that while the physics in solid materials and cold atoms are essentially identical, their physical parameters are chosen in totally different regions. In this work we mainly discuss the physics in cold atoms, thus all the energy parameters are comparable in magnitude [35].

Pairing symmetry.- Firstly, the topology of the Fermi surface is determined by solely the physics at zero momentum. In this case the influence of the Cooper pair momentum $\mathbf{Q}$ can be fully absorbed by defining two effective in-plane Zeeman fields $\bar{h}_{x}=h_{x}-\alpha Q_{y} / 2$, $\bar{h}_{y}=h_{y}+\alpha Q_{x} / 2$ and an effective chemical potential $\bar{\mu}=\mu-E_{Q}$, with $E_{Q}=\left(Q_{x}^{2}+Q_{y}^{2}\right) / 4$ the corresponding kinetic energy of the Cooper pair. For convenience, we also define Zeeman field strength and effective Zeeman field strength as $h^{2}=h_{x}^{2}+h_{y}^{2}+h_{z}^{2}$ and $\bar{h}^{2}=h_{z}^{2}+\bar{h}_{x}^{2}+\bar{h}_{y}^{2}$, respectively. The eigenvectors of the single particle Hamiltonian $H_{0} \phi_{\mathbf{k} s}=\xi_{\mathbf{k} s} \phi_{\mathbf{k} s}$, where $s= \pm$ define the chirality of the bands. $\phi_{\mathbf{k} s}$, hereafter, is called as dressed basis. Assume $\phi_{\mathbf{k}, s}=\left(a_{\mathbf{k} s} c_{\mathbf{k}, \uparrow}+c_{\mathbf{k}, \downarrow}\right) / \sqrt{\left(\left|a_{\mathbf{k} s}\right|^{2}+1\right.}$, with $a_{\mathbf{k} s}=\left(s \bar{h}-h_{z}\right) /\left(\bar{h}_{x}+i \bar{h}_{y}\right)$. To observe the topological phase, the chemical potential should cut only one band, see Fig. 1. In the dressed basis, we find 


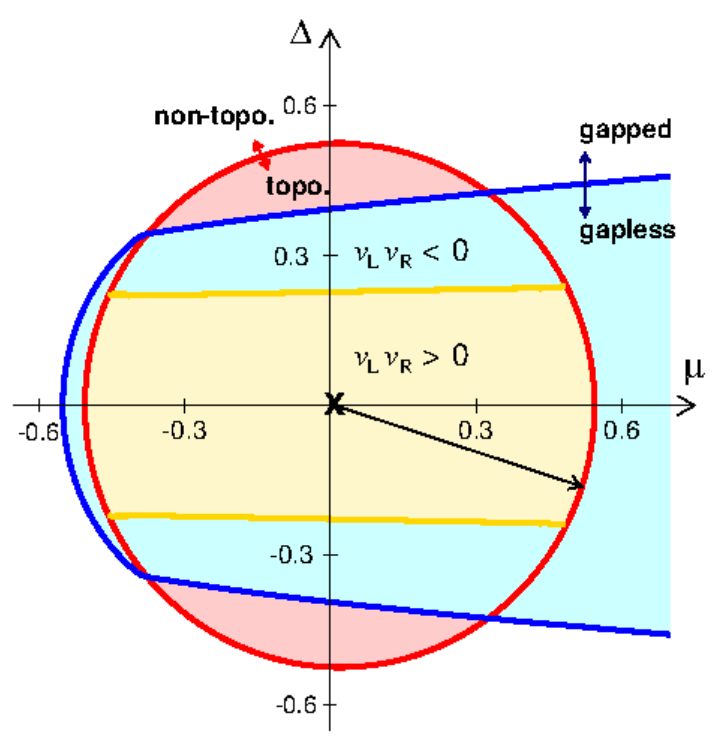

FIG. 3: (Color online). Phase diagram in the $\mu-\Delta$ plane. The solid circle represents the boundary between topological phase and trivial phase, and the open parabola represents the boundary between gapless phase and gapped phase. The overlaps between these two curves define different phases. In the topological gapless phase regime, the (almost) horizontal line define two different phases with counter-propagating $\left(v_{\mathrm{L}} v_{\mathrm{R}}<0\right)$ edge modes and co-propgatating $\left(v_{\mathrm{L}} v_{\mathrm{R}}>0\right)$ edge modes. This phase diagram is obtained using parameters $\alpha=0.5, h_{x}=0.2, h_{y}=0.1, h_{z}=0.5, Q_{x}=0.3, Q_{y}=0.2$.

$\left\langle\phi_{\mathbf{k}+\frac{\mathbf{Q}}{2},-} \phi_{-\mathbf{k}+\frac{\mathbf{Q}}{2},-}\right\rangle=A_{\mathbf{k}, \mathbf{Q}} \Delta$, where $\Delta$ is the $s$-wave pairing strength and the pairing function reads as

$$
A_{\mathbf{k}, \mathbf{Q}}=\frac{a_{\mathbf{k}+\mathbf{Q} / 2-}-a_{-\mathbf{k}+\mathbf{Q} / 2-}}{\sqrt{\left(\left|a_{\mathbf{k}+\mathbf{Q} / 2-}\right|^{2}+1\right)\left(\mid a_{-\mathbf{k}+\mathbf{Q} / 2-\left.\right|^{2}+1}\right.}} .
$$

Notice that the numerator of $A_{\mathbf{k}, \mathbf{Q}}$ is an odd function with respect to $\mathbf{k}$, while the denominator is an even function, thus we have

$$
A_{\mathbf{k}, \mathbf{Q}}=\alpha\left(i k_{x}+\eta k_{y}\right) f\left(k^{2}\right)+\cdots,
$$

where all the even order terms ( $s$-wave and $d$-wave term) are absent, and the suspension point represent the contribution of higher orbital components. Notice that $f\left(k^{2}\right)$, which may be a complex number here, is a function of $k^{2}$, thus a small fraction of higher orbital components have been absorbed into the first term. Eq. 4 is the major observation in this work. We can recover the well-known $k_{x}+i k_{y}$ pairing at the limit without in-plane Zeeman field, in which condition $f\left(k^{2}\right)=1 / \sqrt{h_{z}^{2}+\alpha^{2} k^{2}}$. Obviously, $\eta$ controls the asymmetry of the superfluids. Similar analysis show that all the intra-band couplings should be an odd function thus $p$-wave type, while the inter-band pairing can be $s$-wave type in the small momentum limit, see schematically shown in Fig. 11 The pairing symmetry for other types of SOC and Zeeman field can also be examined in a simialr way.
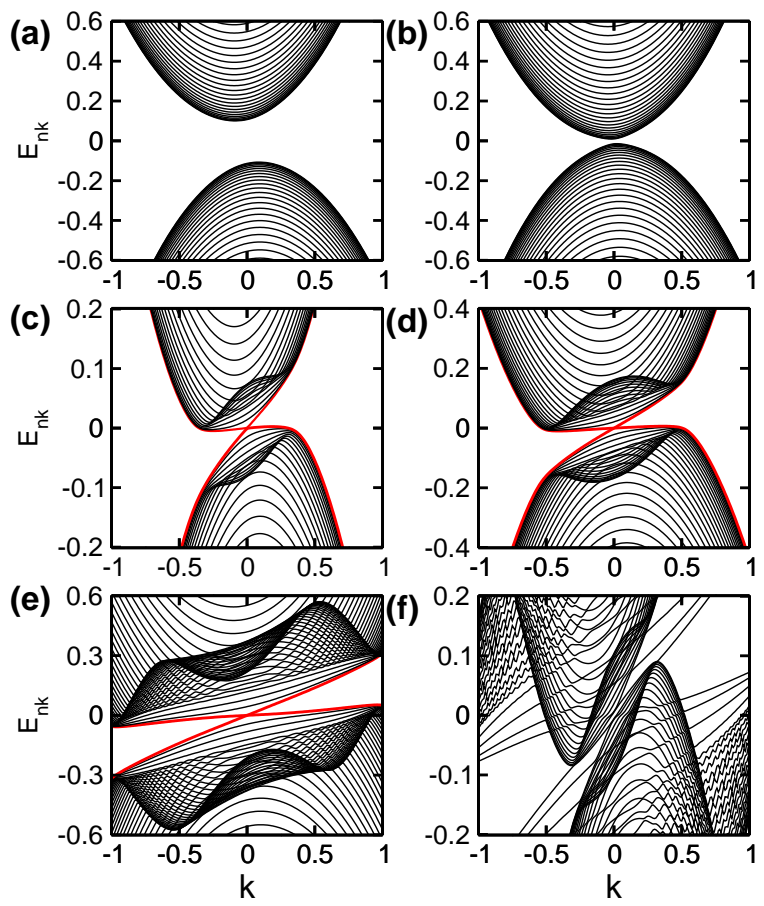

FIG. 4: (Color online). Typical band structure in a stripe geometry with width $L=100$. From (a) - (f), $\mu=-0.6,-0.5$, $-0.4,-0.3,0.3$, and 0.7 , respectively. $\Delta=0.2$, and for vaues of other parameters see Fig. 3] In (c) - (f), the red solid lines represent the edge modes.

We have verified that the first term is always dominant over the second term for the typical parameter regimes in cold atoms. In actually, we can easily check that when $\alpha k \gg \bar{h}, h, A_{\mathbf{k}, \mathbf{Q}} \sim\left(k_{x}+i k_{y}\right) /|\mathbf{k}|$. In this sense, we can conclude that the effective in-plane Zeeman field generally play the role of inducing asymmetry to the effective pairing, which is controlled by $\eta$ in Eq. 4. In Fig. 2a - b we plot the pairing function in momentum space, which show clearly asymmetry due to the presents of in-plane Zeeman field. In Fig. 22-d, we plot the asymmetry factor $\eta$ as a function of in-plane Zeeman field. We see that $|\eta|=1$ only when $\left|\bar{h}_{x}\right|=\left|\bar{h}_{y}\right|$, however, at this condition, its phase still depends strongly on the in-plane Zeeman field. When $\left|\bar{h}_{y}\right| \gg\left|\bar{h}_{x}\right|$, we find $|\eta| \ll 1$, and the superfluids is continuously tuned from $k_{x}+i k_{y}$ superfluids to $k_{y}$ superfluids. Similarly, the $k_{x}$ superfluids can be obtained in the limit when $\left|\bar{h}_{y}\right| \ll\left|\bar{h}_{x}\right|$. Here we need to emphasize that the asymmetry of the superfluids just changes the properties of the superfluids (gapped or gapless, etc); however, it will not change its topology, which is determined solely by the effective Zeeman field strength, see Eq. 5 .

Phase diagram.- The out-of-plane Zeeman field and $\mathrm{SOC}$ is essential to realize a single band with non-trivial Berry phase, which is prerequisite for the realization of topological matters with $s$-wave interactions. The topological phase transition can be determined the Pfaffian 
of the Hamiltonian. We can define a topological index $\mathcal{M}=\operatorname{sign}(\operatorname{Pf}(\Gamma(0)))$, where $\Gamma(\mathbf{k})=-i H_{\mathrm{BdG}}(\mathbf{k}) \Lambda$, and $\Lambda$ is the standard particle-hole symmetry operator without the complex conjugate operator 36 . $\mathcal{M}=-1$ corresponds to topological phase. We determine the topological boundary as

$$
\bar{\mu}^{2}+\Delta^{2}<h_{z}^{2}+\bar{h}_{x}^{2}+\bar{h}_{y}^{2} .
$$

The above result is further verified by Chern number for all the filled bands $\mathcal{C}=\sum_{n} \mathcal{C}_{n}$, where $\mathcal{C}_{n}=$ $-\operatorname{Im} \frac{1}{2 \pi} \int d^{2} k\left\langle\partial_{k_{x}} \psi_{n \mathbf{k}} \mid \partial_{k_{y}} \psi_{n \mathbf{k}}\right\rangle$ and $\left|\psi_{n \mathbf{k}}\right\rangle$ the eigenvalues of the $\mathrm{BdG}$ equation. We find $\mathcal{C}=1$ in the topological regime defined above, and $\mathcal{C}=0$ otherwise. We see that the topological boundary is identical to that for topological BCS superfluids with out-of-plane Zeeman field [4, 31], except that now the Zeeman field should be replaced by the effective Zeeman field. This topological boundary condition is plotted in Fig. 3] by a perfect circle with radius $R=\bar{h}$ (notice that the center of the circle is shifted by the kinetic energy of Cooper pair $E_{Q}$ ). However due to the present of $\mathbf{k} \cdot \mathbf{Q}$ term in the diagonal term in Eq. 1] the system can become gapless at $\mathbf{k} \neq 0$. This is a general feature of the FFLO phase both in solids and cold atoms. The analytical eigenvalues for the original Hamiltonian can not be obtained anymore. The boundary for gappless and gapped phase (defined by $\left.\operatorname{Det}\left(H_{\mathrm{BdG}}(\mathbf{k} \neq \mathbf{0})\right)=0\right)$ is determined numerically in Fig. 3. which is represented by a right open parabola. The different phases in the parameter space thus can be defined by the overlaps between these two curves. Notice that both the topological phase and trivial phase can be either gapped or gapless. Here the gapped FFLO phase is defined as a separate phase because in this regime, the Majorana Fermion can be realized at the vortex core, which is protected by a large fundamental gap. The gapless FFLO phase is not suitable for this particular application, however, it possesses some intriguing features which are beyond the accessibility of its counterpart in solid materials, see below.

Edge modes and finite size effect- It is well-known in condensed matter community that topological gapless edge modes may emergent at the boundary of topological matters due to the bulk-edge correspondence 37. In other words, the edge modes are a manifestation of bulk topology. Such topological protected edge modes have been widely discussed in the context of topological insulators 38 40 and quantum spin Hall effect 41 44, however, direct observation of the topologically protected edge modes are always a great challenge in solid materials. In this sense, it is important to study the topologically protected edge modes in topological BCS and topological FFLO superconductors in ultracold atom systems, which can be directly probed in cold atom systems using momentum resolved radio frequency spectroscopy 45 47.

We present the major results for a stripe geometry along $x$ direction in Fig. 4 for fixed chemical potential.

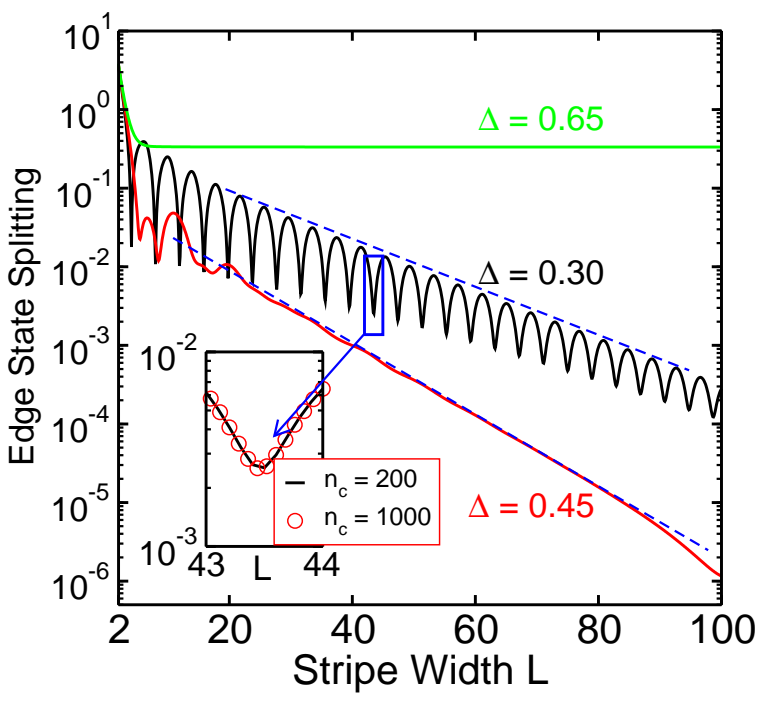

FIG. 5: (Color online). Finite size effect for localized edge modes. $\mu=0.1$ and for the values of other parameters, see Fig. 3 The gap width for trivial gapped phase $(\Delta=0.65)$ is also presented for comparison.

The stripe width along the perpendicular direction is set to $L=100$, which is large enough to ensure that the coupling between the two edges are vanishing small. Hard wall boundary condition is used. We numerically solve the BdG equation using plane wave basis by assuming $\Psi_{k}=e^{i k x} \sum_{n} c_{n} \phi_{n}(y)$, where $\phi_{n}(y)=\sin (n \pi y / L)$, with $n$ smaller than a truncation $n_{c}$. We have verified that the topological edge modes can always be observed in the regime defined by Eq. 5. In the gapless topological FFLO phase regime, the gapless excitations occurs at $k \neq 0$, and the bulk excitation gap is still finite at zero momentum. This finite energy gap ensures that the edge modes can always being directly detected via local momentum resolved radio frequency spectroscopy [45-47].

We can write the edge modes near $k \rightarrow 0$ as

$$
H_{\text {edge }}=\sum_{k} v_{\mathrm{L}} \psi_{\mathrm{L}}^{\dagger} k \psi_{\mathrm{L}}+v_{\mathrm{R}} \psi_{\mathrm{R}}^{\dagger} k \psi_{\mathrm{R}}
$$

where $\mathrm{L}$ and $\mathrm{R}$ correspond to the left and right edge, respectively. We find the gapless topological FFLO phase regime can be divided into two different regimes by the sign of $v_{\mathrm{L}} v_{\mathrm{R}}$, where $v_{\mathrm{L}} v_{\mathrm{R}}>0$ corresponds to the copropagating edge modes while its opposite corresponds to the counter-propagating edge modes, respectively. It represents the most striking feature of this new topological phase. In solid materials, $v_{\mathrm{L}}=-v_{\mathrm{R}}$ is always fulfilled, thus only the counter-propagating edge modes can be observed. In some metarials with Chern number greater than one, co-propagating edge modes may be observed, however, these modes should propagate at the same edge 48. This interesting feature directly comes from the finite momentum pairing and in-plane Zeeman field. We need to emphasize that different velocities cor- 
respond to different density of states (DOS) near zero energy, therefore it can be directly verified in future experiments.

We explore the finite size effect for the same geometry defined above. The edge state splitting as a function of stripe width $L$ is presented in Fig. 5. We can define the superfluids coherence length $\xi_{0}=\hbar v_{\mathrm{F}} / E_{0}$, where $v_{\mathrm{F}}$ and $E_{0}$ are the Fermi velocity and energy gap at zero momentum respectively. In the gapped topological phase regime, we observe that the splitting decay exponentially, $\delta E \sim e^{-L / \xi}$, where $\xi \sim \xi_{0}$. For $\Delta=0.30$, we find $\xi=$ $14.2\left(\xi_{0}=17.7\right)$, and for $\Delta=0.45, \xi=9.0\left(\xi_{0}=11.7\right)$. In the gapless phase regime, the strong couping between different modes near $E=0$ gives rise to oscillation of the edge state splitting, which can be formulated as $\delta E \sim$ $f(L) e^{-L / \xi}$, where $f(L)$ is an nonzero oscillation function (see inset of Fig. [5 for different truncations $n_{c}$ ). Such oscillation should be a typical feature of edge modes in gapless topological phase. In the trivial phase regime, no edge modes can be observed, and the gap splitting quickly approaches the bulk gap when $L>\xi_{0}$ (here for $\left.\Delta=0.65, \xi_{0}=6.3\right)$.

To conclude, in this work we have discussed the basic physics in topological FFLO superfluids which still lack a direct counterpart in solid materials. The novel features of this new phase can be directly implemented in state-of-the-art ultracold atoms in a tunable manner, including not only interaction strength, but also pairing asymmetry. These investigations can greatly enrich our understanding of topological matter and its basic pairing symmetry - a fundamental issue in modern physics.

Acknowledgement: This work is supported by Hong Kong RGC/GRF Projects (No. 401011 and No. 2130352) and the Chinese University of Hong Kong (CUHK) Focused Investments Scheme.

* Electronic address: skylark.gong@gmail.com

[1] Y.-J. Lin, K. Jiménez-García and I. B. Spielman, Nature 471, 83 (2011).

[2] Pengjun Wang, Zeng-Qiang Yu, Zhengkun Fu, Jiao Miao, Lianghui Huang, Shijie Chai, Hui Zhai, and Jing Zhang Phys. Rev. Lett. 109, 095301 (2012).

[3] Lawrence W. Cheuk, Ariel T. Sommer, Zoran Hadzibabic, Tarik Yefsah, Waseem S. Bakr, and Martin W. Zwierlein Phys. Rev. Lett. 109, 095302 (2012).

[4] Ming Gong, Sumanta Tewari, and Chuanwei Zhang, Phys. Rev. Lett. 107, 195303 (2011).

[5] Li Han and C. A. R. Sá de Melo, Phys. Rev. A 85, 011606 (2012).

[6] Kangjun Seo, Li Han, and C. A. R. Sá de Melo, Phys. Rev. A 85, 033601 (2012).

[7] Zeng-Qiang Yu and Hui Zhai, Phys. Rev. Lett. 107, 195305 (2011).

[8] Jing Zhou, Wei Zhang, and Wei Yi, Phys. Rev. A 84, 063603 (2011).
[9] Lei Jiang, Xia-Ji Liu, Hui Hu, and Han Pu, Phys. Rev. A 84, 063618 (2011).

[10] Fan Wu, Guang-Can Guo, Wei Zhang, and Wei Yi, Phys. Rev. Lett. 110, 110401 (2013).

[11] Zhen Zheng, Ming Gong, Xubo Zou, Chuanwei Zhang, and Guangcan Guo, Phys. Rev. A 87, 031602 (2013).

[12] Zhen Zheng, Ming Gong, Yichao Zhang, Xubo Zou, Chuanwei Zhang, Guangcan Guo, arXiv:1212.6826v2.

[13] Gang Chen, Ming Gong, and Chuanwei Zhang, Phys. Rev. A 85, 013601 (2012).

[14] Chunlei Qu, Zhen Zheng, Ming Gong, Yong Xu, Li Mao, Xubo Zou, Guangcan Guo and Chuanwei Zhang, Nature Communications 4, 3710 (2013).

[15] Wei Zhang and Wei Yi, Nature Communications 4, 3710 (2013).

[16] Xia-Ji Liu and Hui Hu, Phys. Rev. A 88, 023622 (2013).

[17] Chun Chen, Phys. Rev. Lett. 111, 235302 (2013).

[18] Ming Gong, Gang Chen, Suotang Jia, and Chuanwei Zhang, Phys. Rev. Lett. 109, 105302 (2012).

[19] Renyuan Liao, Yu Yi-Xiang, and Wu-Ming Liu, Phys. Rev. Lett. 108, 080406 (2012).

[20] Hui Hu and Xia-Ji Liu, New J. Phys. 15, 093037 (2013).

[21] Lianyi He, Xu-Guang Huang, Hui Hu, and Xia-Ji Liu, Phys. Rev. A 87, 053616 (2013).

[22] Renyuan Liao, Yu Yi-Xiang, and Wu-Ming Liu, Phys. Rev. Lett. 108, 080406 (2012).

[23] Kangjun Seo, Chuanwei Zhang, and Sumanta Tewari, Phys. Rev. A 87, 063618 (2013).

[24] Ran Wei and Erich J. Mueller, Phys. Rev. A 86, 063604 (2012).

[25] Di Xiao, Ming-Che Chang, and Qian Niu, Rev. Mod. Phys. 82, 1959 (2010).

[26] Xiao-Liang Qi and Shou-Cheng Zhang, Rev. Mod. Phys. 83, 1057 (2011).

[27] M. Z. Hasan and C. L. Kane, Rev. Mod. Phys. 82, 3045 (2010).

[28] Chuanwei Zhang, Sumanta Tewari, Roman M. Lutchyn, and S. Das Sarma, Phys. Rev. Lett. 101, 160401 (2008).

[29] Masatoshi Sato, Yoshiro Takahashi, and Satoshi Fujimoto, Phys. Rev. Lett. 103, 020401 (2009).

[30] Lev P. Gor'kov, Emmanuel I. Rashba, Phys. Rev. Lett. 87, 037004 (2001).

[31] J. Alicea, Rep. Prog. Phys. 75, 076501 (2012).

[32] Ming Gong et al (unpublished).

[33] B. S. Chandrasekhar, Appl. Phys. Lett. 1, 7 (1962).

[34] A. M. Clogston, Phys. Rev. Lett. 9, 266 (1962).

[35] In solid materials, the Zeeman field and SOC energy are generally much smaller than the chemical potential (or Fermi energy).

[36] Parag Ghosh, Jay D. Sau, Sumanta Tewari, and S. Das Sarma, Phys. Rev. B 82, 184525 (2010).

[37] Y. Hatsugai, Phys. Rev. Lett. 71, 3697 (1993).

[38] Wen-Yu Shan, Hai-Zhou Lu and Shun-Qing Shen, New J. Phys. 12, 043048 (2010).

[39] Bin Zhou, Hai-Zhou Lu, Rui-Lin Chu, Shun-Qing Shen, and Qian Niu Phys. Rev. Lett., 101, 246807 (2008).

[40] Jacob Linder1, Takehito Yokoyama2, and Asle Sudbo, Phys. Rev. B 80, 205401 (2009).

[41] C.L. Kane and E.J. Mele, Phys. Rev. Lett. 95, 226801 (2005).

[42] B.A. Bernevig and S.C. Zhang, Phys. Rev. Lett. 96, 106802 (2006).

[43] C.L. Kane and E.J. Mele, Phys. Rev. Lett. 95, 146802 (2005). 
[44] B.A. Bernevig, T.L. Hughes and S.C. Zhang, Science 314, 1757 (2006).

[45] J. T. Stewart, J. P. Gaebler, D. S. Jin, Nature 454, 744 (2008)

[46] Qijin Chen, and K. Levin, Phys. Rev. Lett. 102, 190402 (2009).
[47] Hui Hu, Lei Jiang, Xia-Ji Liu, and Han Pu, Phys. Rev. Lett. 107, 195304 (2011).

[48] Netanel H. Lindner, Gil Refael, and Victor Galitski, Nat. Phys. 7, 490 (2011). 